\title{
Implementation of a Hyperspectral X-ray Camera control and processing software chain
}

\author{
F. Van Assche $,{ }^{a}, 1$ S. Vanheule, ${ }^{a}$ S. Cipiccia, ${ }^{b}$ L. Vincze,${ }^{c}$ L. Van Hoorebeke, ${ }^{a}$ and \\ M. N. Boone ${ }^{a}$ \\ ${ }^{a}$ Radiation Physics group, UGCT, Department of Physics and Astronomy, Ghent University, \\ Proeftuinstraat 86 (N12), B-9000 Ghent, Belgium \\ ${ }^{b}$ Diamond Light Source, \\ Harwell Science and Innovation Campus, Didcot OX11 0QX, United Kingdom \\ ${ }^{c} X$-ray Microspectroscopy and Imaging group (XMI), Department of Chemistry, Ghent University, \\ Krijgslaan 281 (S12), B-9000 Ghent, Belgium \\ E-mail: frederic.vanassche@ugent.be
}

ABSTRACT: Hyperspectral X-ray detectors, which provide the full energy spectrum detected by each individual pixel, have become available for use in lab-based facilities. The addition of spectral information currently comes at the cost of low overall acceptable flux rates, and can introduce countrate nonlinearity at higher energies. Neither of these drawbacks are desirable for transmission imaging and tomography. In this paper a new data processing software chain is presented for the SLcam, a pnCCD-based hyperspectral camera prototype, allowing for more control over the conversion from raw frames to hyperspectral images. Complementary to the processing software, a set of live data visualisations was developed to aid in monitoring ongoing experiments and to allow for preliminary data processing on-the-fly. The combination of these software elements forms the first step towards general applicability of hyperspectral imaging at laboratory tomography setups.

Keywords: X-ray detectors; Solid state detectors; Detector control systems; Data acquisition concepts

\footnotetext{
${ }^{1}$ Corresponding author.
} 


\section{Contents}

1 Introduction 1

1.1 Hyperspectral imaging 1

1.2 The color X-ray camera 1

1.3 New software suite 2

2 Software processing chain 3

2.1 General features 3

2.2 Frame processing basics 4

2.3 Multiple simultaneous datastreams 5

2.4 A note on performance 5

3 Graphical representations $\quad 5$

3.1 Energy centroid view $\quad 6$

$\begin{array}{lll}3.2 & \text { False-colour view } & 7\end{array}$

$\begin{array}{llr}4 & \text { Summary } & 8\end{array}$

\section{Introduction}

\subsection{Hyperspectral imaging}

Recent advances in detector technology have made hyperspectral X-ray detectors avaiable for wider applications. These cameras provide the full detected X-ray energy spectrum for every individual pixel, typically using on the order of one to several thousand narrow energy bins. With these systems the use of full field spectral imaging has come within reach of the average lab-based facility.

The switch to energy dispersive imaging is however not without drawbacks. Because spectral detectors need to measure the energy of each photon individually, proper detection is only possible when there is minimal temporal and spatial overlap between photons. This presents a fundamental limit to the flux rates that can be handled by a hyperspectral system. The current reality of this is that hyperspectral imaging requires far longer exposure times than conventional integrating systems, and is by consequence rarely used for transmission imaging and tomography.

\subsection{The color X-ray camera}

One such system available at the Ghent University Centre for X-ray Tomography (UGCT) is the SLcam [1]. This camera is based on a pnCCD with a fully depleted $450 \mu \mathrm{m}$ thick silicon active volume, and 264 by 264 pixels of $48 \mu \mathrm{m}$ pixel pitch. The sensor is coupled to $11 \mathrm{bit}$ ADCs through front-end electronics with exceptionally low noise characteristics [2], enabling the camera to reach an energy resolution of $147 \mathrm{eV}$ around the $\mathrm{Mn} \mathrm{K} \alpha$ peak. This performance is comparable to state-of-the-art single-pixel energy dispersive detectors. 
The SLcam is subject to the aforementioned common issues seen in hyperspectral detectors. These drawbacks follow from the operating principle of the camera and the physical constraints of the system. To measure the energy of incident photons these have to be detected and reconstructed individually, and any full or partial spatial overlap of photons within a single frame makes this energy reconstruction difficult to impossible. As a rule of thumb, no more than $10 \%$ of a single frame can be occupied by photon induced charges before the average amount of overlapping charge clusters becomes too large. The SLcam has a framerate of $400 \mathrm{~Hz}$, around 70000 pixels, and an active area of $264 \times 264 \times(48 \mu \mathrm{m})^{2}=1.6 \mathrm{~cm}^{2}$. On average photons generate charge clusters around four pixels in size. Given these numbers the flux rate should never exceed about $4.4 \times 10^{5} \mathrm{~cm}^{-2} \mathrm{~s}^{-1}$, which is very low even for laboratory high-resolution transmission imaging and tomography work.

Coupled to the low flux limitation is the issue of countrate nonlinearity [3]. Because of the relatively small pixel size of $48 \mu \mathrm{m}$, nearly all photons generate a charge cloud that is bigger than one individual pixel. This charge sharing effect is energy dependent, which means that charge clusters tend to get bigger as photon energies increase. This not only causes the maximum flux rates to be lower at higher energies, but also means that after transmission through a sample, energy-dependent countrates detected in each pixel will be influenced by the full photon spectrum seen by that pixel, as demonstrated in [4].

To gather sufficient statistics at these low count rates, transmission radiographies are typically taken using exposure times of half an hour of more. A full tomography scan usually collects a comparable number of projections as the detector's horizontal pixel count. In case of the SLcam this requires collecting around 260 radiographies for a total scanning time of $260 \times 30 \mathrm{~min}=130 \mathrm{~h}$, or roughly five and a half days. Both detector stability and sample stability must be guaranteed for a full scan to complete successfully. While better processing algorithms and more stable control software do not directly alleviate the long scanning times, they do enable the extraction of more information from the gathered frames through parallel specialised processing chains and enhanced photon pile-up correction. This helps make better use of the limited number of collected photons over workable expore times.

\subsection{New software suite}

A completely new software suite was developed for the SLcam in an attempt to deal with these shortcomings within the context of transmission tomography. On top of the general problems presented in 1.2, a few device and application specific wishes were addressed in the development of the new software chain.

Relatively lacking long-term stability of the previous software combined with incomplete handling of hardware safety compromising faults led to poor reliability of the camera for scans lasting a week or more. The hardware control software components have been rewritten to address these deficiencies and make up a large part of the total software suite, but will not be discussed in detail. These hardware control and monitoring functions are performed by the "housekeeper" application included in the software architecture overview in figure 1.

Acquiring more control over the processing between raw detector frames and final output datasets was a second major goal in the development of the new software suite. This has been achieved through the modular design of the new acquisition chain as will be elaborated in the following sections. 


\section{Software processing chain}

\subsection{General features}

The new image acquisition and processing software chain follows a highly modular and flexible approach. This is enabled thanks to the use of the ZeroMQ library, a high-performance asynchronous distributed messaging solution. Figure 1 shows a schematical representation of the normal data flow. Each block represents an isolated process, written in the form of a simple separate $\mathrm{C}$ or $\mathrm{C}++$ binary. The "ADCs" block is the exception, implemented as a Linux kernel driver, which performs the actual interrupt-driven ADC hardware readout and pushes the raw frames on a ringbuffer for the "grabber" userspace process to read and broadcast using ZeroMQ. Communication between ZeroMQ-based processes is completely network transparent and inherently parallel, which presents a number of advantages:

- Performance is not limited by a single computing node and scales in function of the number of worker processes. These workers can be started and stopped at will without dropped frames.

- Each separate element can be run on different nodes on the network if so desired, allowing for rapid scaling of performance for more complex algorithms.

- Data can be extracted at any point along the chain for intermediate storage, analysis, debugging, calibration, ...

- Extracted or generated data can be injected into the system again for later processing. This enables easy storage of raw frames of interest for reprocessing at a later time, e.g. for more detailed analysis of unique datasets.

- Multiple independent processing flows can run off the same feed of raw frames enabling simultaneous output of specialised or experiment specific datasets together with the normal general purpose processing.

- All tools and graphical interfaces can run independently over the regular network, allowing multiple operators to work together on their own workstations.

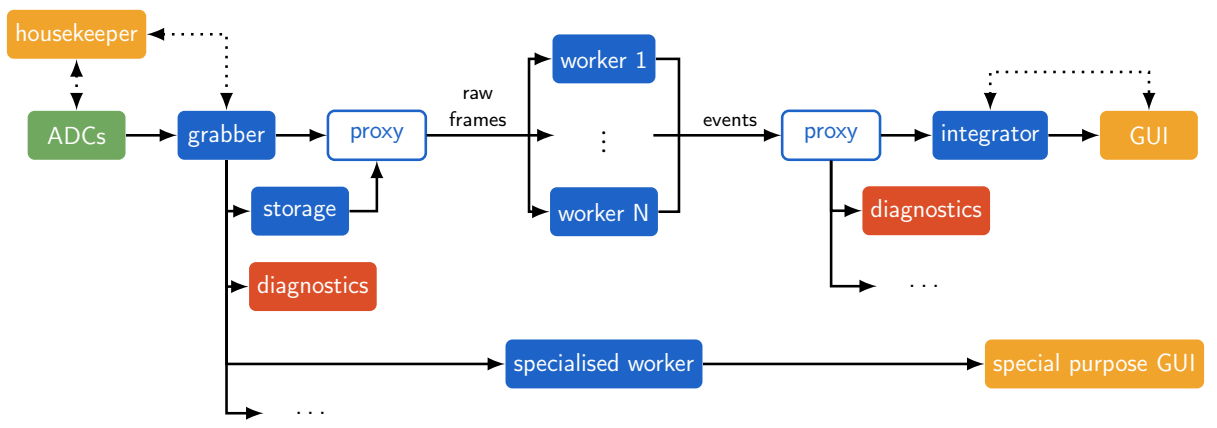

Figure 1: Schematic representation of an example configuration using the new data flow system. The blocks represent isolated processes, all communicating using ZeroMQ message streams. Solid lines represent image and sensor data, dotted lines indicate control and metadata streams. 


\subsection{Frame processing basics}

Photon counting detectors with pixel sizes on the order of a few tens of $\mu \mathrm{m}$ or smaller all experience so called charge sharing [5]. This phenomenon occurs when the detector geometry and electronhole transport parameters in the active material are such that charge clusters generated by incident photons frequently occupy a volume bigger than a single pixel. The charge then ends up being collected by multiple neighbouring pixels, and proper energy measurement of detected photons needs to take this effect into account. The basic charge cluster reconstruction algorithm loops over every raw frame in search of pixels with charges above a noise suppression threshold, and each set of connected neighbouring pixels above threshold is marked as a candidate event. The total charge of the event is collected, and a center-of-mass is calculated using the pixel positions weighted by their respective detected charges. This information is finally stored as an event with total energy, center-of-mass position and a set of diagnostic parameters.

Because the energy and center-of-mass position of an event is calculated and stored as floating point values, these can be filtered and binned independently of the detector's pixel count and ADC precision. The hardware uses 528 separate analog gain and filtering stages (two per column of pixels) and four ADCs (one per sensor quadrant). Minor differences in the actual gains achieved by these 528 combinations, combined with pixel-wise dark current corrections, slightly spreads out the ADC bins for each pixel. This means the energy can be rebinned with more precision than the raw $\mathrm{ADC}$ bins for a small improvement in energy reconstruction.

A similar principle applies to the position values. Since the large majority of events are seen by more than one pixel, the center-of-mass position carries localisation information at a finer pitch than the physical pixel grid. By rebinning the $x$ and $y$ coordinates not to the physical 264 by 264 grid, but for example on a 1000 by 1000 grid, an image using a virtual pixel size of $12.7 \mu \mathrm{m}$ can be obtained [6]. Figure 2a shows a radiograph of the head of a horsefly binned to the physical pixel grid. This same object imaged using $4 \times 4$ subsampling is displayed in figure $2 \mathrm{~b}$, with a virtual pixel size of $12 \mu \mathrm{m}$. The images are the result of one five minute acquisition processed in parallel with and without subsampling, leading to a 16-fold increase in noise levels in the subsampled image.

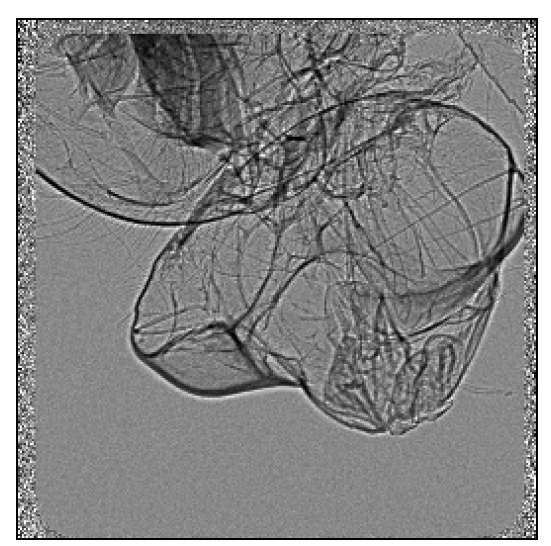

(a) Imaged without subsampling.

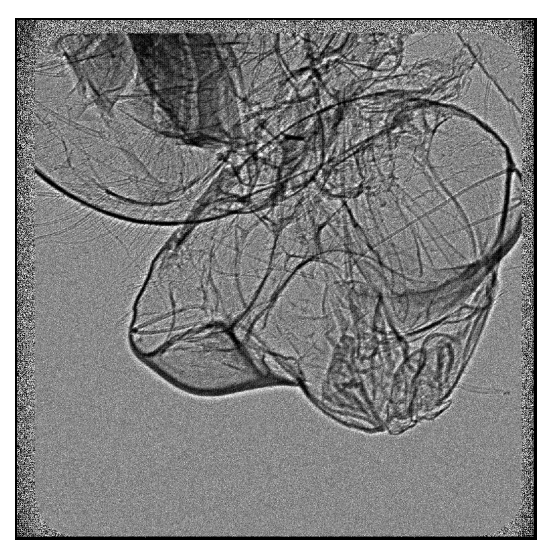

(b) Imaged using $4 \times 4$ subsampling.

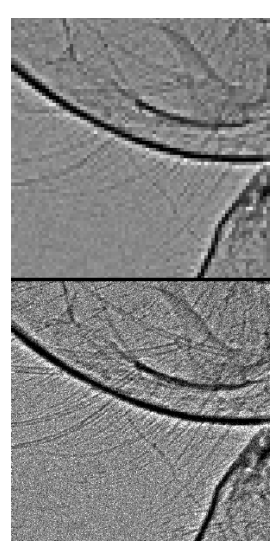

(c) Detail view.

Figure 2: Demonstration of subsampling to achieve virtual pixel sizes smaller than the physical $48 \mu \mathrm{m}$ pixels. Tube parameters: $30 \mathrm{keV}$, tungsten target, and a flux of $1.9 \times 10^{5} \mathrm{~cm}^{-2} \mathrm{~s}^{-1}$. 


\subsection{Multiple simultaneous datastreams}

As described in section 2.1, the acquisition chain is able to run multiple processing workflows simultaneously. By using different sets of calibration datasets, cluster reconstruction algorithms, event filters, energy and spatial binning settings, and other tuneable detector parameters, specialised processing modes can be developed. For example, by using $2 \times 2$ subsampling and a clustering algorithm focused on pile-up correction and count rate linearity, a processing mode focused on tomography can be included. Operating this mode in tandem with one specialising in the best possible energy resolution, the extra flexibility offered by hyperspectral detectors can be leveraged.

One could then use the tomography-specialised dataset to extract the 3D morphology of a sample at high quality. The energy-focused dataset then provides spectral information useful for material identification, which can be spatially constrained using the morphology data obtained from the tomography reconstruction. This way exploitation of hyperspectral transmission imaging data becomes possible beyond simple subtractive edge-imaging. These specialised processing modes do not have to be limited to just improvements in spatial or energy resolution. If experimental conditions are sufficiently well constrained, highly specialised datasets can be produced on-the-fly. These can all be flexibly integrated next to the normal data flow.

While this multi-stream appproach is not able to completely alleviate the issues fundamental to hyperspectral detectors, gaining more information from a single acquisition can help cope with these problems. By having different views of the same data available, it becomes easier to identify problematic data or experimental conditions.

\subsection{A note on performance}

The present combination of hardware readout, processing algorithms, data storage and visualisation is able to run very comfortably on any modern workstation. At a framerate of $400 \mathrm{~Hz}, 264$ by 264 pixels and 16 bit values per pixel, the stream of raw frames coming from the SLcam requires a bandwidth of slightly under $0.5 \mathrm{GBit} / \mathrm{s}$. This is well within the capabilities of an ordinary workstation for real-time acquisition.

The main limitation at this point occurs when it is desired to write raw frames to storage for later reprocessing. If one would for example store all frames gathered during a typical beamtime, storage requirements easily run into the tens of gigabytes. Doing this in a cost-effective manner mostly restricts storage options to traditional rotating disk hard drives. The speeds achieved by a modest multi-disk setup in practice are on the order of $350 \mathrm{MByte} / \mathrm{s}$ to $400 \mathrm{MByte} / \mathrm{s}$. This would speed up reprocessing of a stored set of frames only about sevenfold compared to live processing, and is currently the main bottleneck of the acquisition software chain.

Because of the distributed and network-transparent software configuration, storage and processing power limitations can easily be overcome if so desired. Even so, the requirements of the current implementation leave plenty of headroom for more involved live data handling.

\section{Graphical representations}

To aid in qualitative observation of live experimental data, two new graphical representations have been included in the software. 


\subsection{Energy centroid view}

The first new representation is called the energy centroid view. This view selects all photons with an energy inside a configurable range, using which the mean energy and its standard deviation are calculated for each pixel. After integrating over a configurable timespan, this 2D set of values is then represented graphically in the user interface as two images by mapping the mean and standard deviation to a color scale. To avoid confusion with intensity-dependent images, the use of a perceptually uniform color scale (e.g. Viridis) is recommended.

The main advantage of this representation lies in its independence from actual photon counts. Areas with identical average photon energy but different photon counts due to absorption or beam intensity variations will still show up as a uniform colour. This is illustrated in figure 3 , showing the spectral and spatial features of the beam at beamline I13-2, Diamond Light Source when using the multilayer monochromator (MLM) with a $\left[\mathrm{Ru}, \mathrm{B}_{4} \mathrm{C}\right]$ strip (100 layers, $4.588 \mathrm{~nm}$ spacing). This type of monochromator introduces a modulation of the intensity of the beam [7], visible in figure 3a. To investigate whether these intensity variations also appear as variations in energy, the SLcam was used during a beam diagnostics beamtime. Figure $3 \mathrm{~b}$ shows the spatial profile of the mean energy of the beam, centered around the $12 \mathrm{keV}$ fundamental spectral peak of the MLM. Because the central value of a peak is measureable with far more precision than the $147 \mathrm{eV}$ SLcam energy resolution, thanks to averaging over a great number of photons, this representation provides proof that the energy profile of beam at I13-2 is uniform after monochromation using the MLM to within $21 \mathrm{eV}$. This high sensitivity of the centroid view is immediately evident from figure $3 \mathrm{c}$, where the same image as in $3 \mathrm{~b}$ was generated using a slightly mismatched detector calibration. Because small variations on the order of a few eV show up clearly in the energy centroid view, it is also often used as a quick verification of detector performance using a source with known spectrum, e.g. an $\mathrm{Fe}^{55}$ radioactive source.

While the energy centroid view may be considered independent of actual photon counts, they will still have an influence on the statistics and reliability of the calculated mean energy. For this reason it is useful to also take the standard deviation representation into account to get an idea of the quality of the energy centroid view in its mean energy representation.

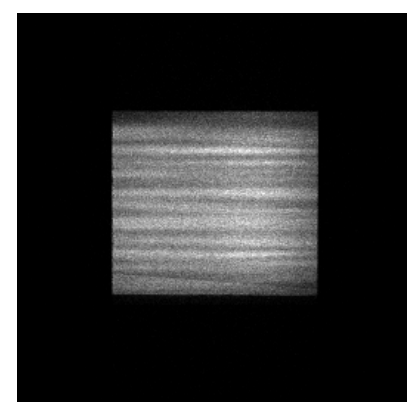

(a) Traditional integrated image.

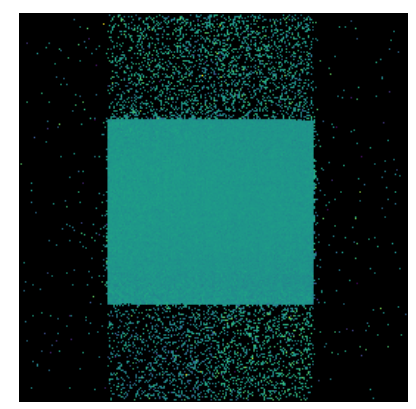

(b) Energy centroid view between $11.5 \mathrm{keV}$ and $12.5 \mathrm{keV}$.

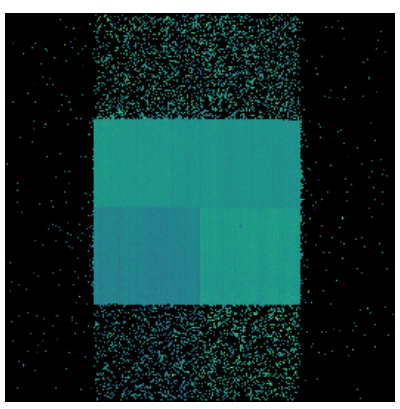

(c) Identical settings with incorrect detector calibration.

Figure 3: Demonstration of the energy centroid view in a $1 \mathrm{keV}$ window around the $12 \mathrm{keV}$ fundamental of the MLM at DLS beamline I13-2, using the 5th beam harmonic and a Si mirror. Sensitivity of this view to small errors in detector calibration is also shown in the rightmost panel. 


\subsection{False-colour view}

The second new representation is called the false-colour view. With this view it is possible to observe both intensity variations as well as spectral information. The images are created by assigning an energy range to each of the three colour planes. Over a configurable integration time, photons are collected for each of the three ranges and the RGB colour planes are filled according to observed photon counts. A simple autoranging function is then applied separately to each of the planes, scaling the 8 bit value range of each colour to the minimum and maximum photon count detected in each of the energy ranges. This representation enables analysis of the spatial distribution of spectral features.

The autoscaling feature is especially useful in cases where it is desired to localise a low flux of photons with a certain energy of interest against a high-flux background of different energy, as illustrated in figure 4. This figure contains data taken at the I13-2 imaging beamline, Diamond Light Source, during a beam diagnostics beamtime using the SLcam. During acquisition a spectral peak around the $17.5 \mathrm{keV}$ molybdenum $\mathrm{K}_{\alpha}$ energy was noticed, which can be seen as the green haze in figure $4 \mathrm{~b}$. These photons are not a direct component of the beam, but are the result of higher energy beam components hitting the molybdenum radiation shields inside the detector, sending out a uniform glow of characteristic molybdenum fluorescent light. This faint component can be identified against the much stronger direct beam using the false-colour view. Also visible using this representation in figure $4 \mathrm{~b}$ is the difference in spatial intensity profile for the MLM fundamental and first harmonic spectral peaks, shown respectively in red and blue.

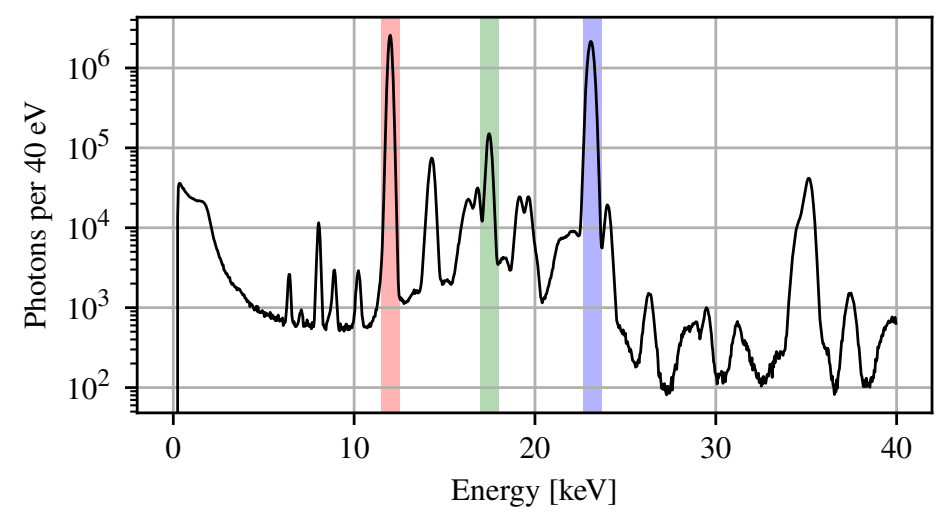

(a) Measured spectrum.

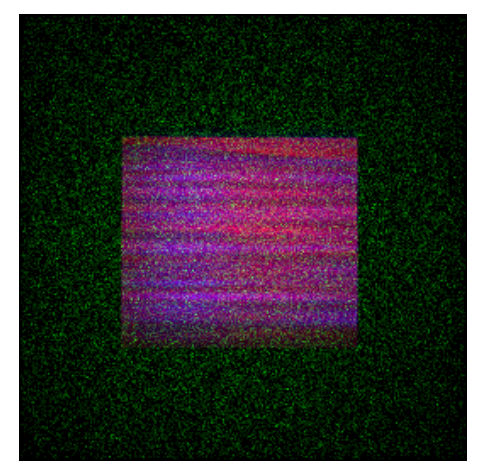

(b) RGB view.

Figure 4: Demonstration of the false-colour view using data from the DLS beamline I13-2. The MLM was used with a Si mirror and a $12 \mathrm{keV}$ monochromator fundamental centered on the 5 th beam harmonic. For the RGB view, the red range represents the $12 \mathrm{keV}$ fundamental energy, the blue range shows the $23.1 \mathrm{keV}$ harmonic, and the green range contains the $17.5 \mathrm{keV} \mathrm{Mo} \mathrm{K}_{\alpha}$ peak. Each of the energy ranges is $1 \mathrm{keV}$ wide, centered around the energy of interest, and is also highlighted on the measured spectrum. 


\section{Summary}

We presented a new, distributed software chain for acquisition and live processing of hyperspectral data obtained using an SLcam energy dispersive X-ray camera. This new software suite allows for more reliable detector operation and provides a wealth of diagnostic and experimental variables not available before. Experiment-specific workflows can now be easily implemented, and the toolset to identify and cope with the current shortcomings of hyperspectral imaging is available.

As part of a graphical user interface two complementary data representations were developed, as presented in this work. These allow for quick exploitation of the spectral information provided by the SLcam in various experimental environments.

\section{Acknowledgments}

Diamond Light Source is acknowledged for the funding and beamtime provided under proposal MT19579. The scientist at the DLS imaging beamline I13-2 are thanked for graceously providing their expertise and assistance during the beamtime. The datasets acquired have been of great help while validating software and detector performance.

This research is funded by the Research Foundation Flanders (FWO) under grant number G0A0417N.

\section{References}

[1] I. Ordavo, S. Ihle, V. Arkadiev, O. Scharf, H. Soltau, A. Bjeoumikhov et al., A new pnCCD-based color $X$-ray camera for fast spatial and energy-resolved measurements, Nucl. Instrum. Meth. A 654 (2011) $250-257$.

[2] S. Herrmann, W. Buttler, R. Hartmann, N. Meidinger, M. Porro and L. Strueder, CAMEX readout ASICs for pnCCDs, in 2008 IEEE Nuclear Science Symposium Conference Record, pp. 2952-2957, Oct, 2008. DOI.

[3] M. N. Boone, New imaging modalities in high resolution X-ray tomography. $\mathrm{PhD}$ thesis, Ghent University, 2013.

[4] M. N. Boone, J. Garrevoet, P. Tack, O. Scharf, D. P. Cormode, D. V. Loo et al., High spectral and spatial resolution $X$-ray transmission radiography and tomography using a Color X-ray Camera, Nucl. Instrum. Meth. A 735 (2014) 644 - 648.

[5] N. Kimmel, Analysis of the charge collection process in solid state X-ray detectors. PhD thesis, Universität Siegen, 2009.

[6] S. Cartier, M. Kagias, A. Bergamaschi, Z. Wang, R. Dinapoli, A. Mozzanica et al., Micrometer-resolution imaging using MÖNCH: towards $G_{2}$-less grating interferometry, Journal of Synchrotron Radiation 23 (Nov, 2016) 1462-1473.

[7] R. Dietsch, A. Rack, T. Weitkamp, M. Riotte, T. Rack, T. Holz et al., Performance of Multilayer Monochromators for Hard X-Ray Imaging with Coherent Synchrotron Radiation, AIP Conference Proceedings 1365 (2011) 77-80. 